\title{
Aproximación a las prácticas ciudadanas en la escuela: el caso del colegio Fernando González Ochoa de la localidad de Usme (Bogotá, Colombia) ${ }^{*}$
}

\section{Approach to citizenship practices at school: the case of the Fernando González Ochoa school in the district of Usme (Bogotá, Colombia)}

\author{
Rodrigo Gómez Pineda \\ Magíster en Comunicación, Desarrollo y Cambio Social de la Universidad Santo Tomás. \\ Historiador egresado de la Universidad Nacional de Colombia. Docente \\ del Colegio Fernando González Ochoa de la Secretaría de Educación de Bogotá D. C. (Colombia) \\ Correo electrónico: rodrigomezpi@yahoo.es
}

Artículo de investigación

DOI: http://dx.doi.org/10.15332/s2339-3688.2017.0001.04

Fecha de recepción: Julio 31 de 2017 • Fecha de aceptación: Diciembre 13 de 2017

* Este trabajo es un extracto de la investigación Aproximación a las prácticas ciudadanas en la escuela: el caso del colegio Fernando González Ochoa de la localidad de Usme, desarrollado entre 2015 y 2016 como trabajo de grado de la maestría en Comunicación, Desarrollo y Cambio Social de la Universidad Santo Tomás de Bogotá. 


\section{Resumen}

En el presente estudio se hace una aproximación a la comprensión de las prácticas ciudadanas escolares de los estudiantes de noveno grado, jornada tarde, del colegio Fernando González Ochoa, ubicado en la localidad quinta de Bogotá (Colombia). Metodológicamente se fundamenta en un estudio cualitativo con enfoque hermenéutico-interpretativo, a través de técnicas como la observación participante, entrevistas semiestructuradas y grupos focales. Se identifican como principales dimensiones de las prácticas ciudadanas escolares: la participación, la convivencia, el sentido de pertenencia, la identidad, el reconocimiento de sí mismo y de los otros, los derechos y las responsabilidades dentro de lo que implica ser joven hoy en el contexto social, cultural, político, económico y educativo del sector de Usme, así como de sectores vulnerables de la ciudad. Esta población juvenil en contexto escolar actúa de acuerdo con su realidad inmediata, siendo permeados por los medios de comunicación, además de la familia y sus pares, que les permite establecer nuevas formas de interacción, diferenciándolos de los adultos y son una muestra de las nuevas maneras de interrelación, comunicación y de participación ciudadana.

Palabras clave: ciudadanía, cultura escolar, juventud, participación.

\section{Abstract}

In the present study, an approximation is made to the understanding of the citizen practices of students in ninth grade at Fernando González Ochoa school, located in the fifth locality of Bogotá (Colombia). Methodologically, it is based on a qualitative study with anhermeneuticinterpretative approach, through research techniques such as participant observation, semistructured interviews and focus groups. The following are identified as the main dimensions of school citizen practices: participation, coexistence, sense of belonging, identity, selfrecognition and recognition of the others, rights and responsibilities within what implies being young today in social, cultural, political, economic and educationalcontext, all of this inside the locality of Usme, as well as in another vulnerable sectors of the city. In a school context, this youth population acts according to their immediate reality, in which they are permeated by the media, as well as by their family and their peers, this allows them to establish new forms of interaction, making a difference between adults and them. All these are a sample of the new ways of interrelation, communication and citizen participation.

Keywords: Citizenship, school culture, youth, participation. 


\section{INTRODUCCIÓN}

La presente investigación pretende entender la ciudadanía y las prácticas ciudadanas de los jóvenes, a partir de sus propias experiencias o vivencias y desde la observación y análisis del investigador dentro del contexto escolar; dado que las lógicas de participación y las actitudes de los jóvenes en relación con su entorno físico, con las personas de su contexto sociocultural y con los grupos a los cuales pertenecen, se manifiestan a través de comportamientos, prácticas, conductas y nuevos lenguajes; y estos no siempre son entendidos por los adultos o son valorados de manera negativa debido a su desconocimiento. El abordaje de la investigación se inicia con la aproximación conceptual, en este caso sobre ciudadanía, cultura escolar, ciudadanía juvenil y juventud; posteriormente se establece una aproximación a las prácticas ciudadanas en la escuela: el caso del colegio Fernando González Ochoa de la localidad de Usme.

La ciudadanía es un tema sobre el cual se ha investigado desde diferentes referentes teóricos, desde distintos periodos históricos y teniendo en cuenta diversos contextos sociales, políticos y culturales, lo cual se hace evidente en la abundante y diversa literatura existente sobre el tema. Por su parte, la ciudadanía juvenil cobra especial interés a partir de la década de 1990, tanto desde la política pública como desde diversas instituciones académicas, de carácter público como privado, debido a la revaloración del ser joven que se presenta desde el fin de la segunda guerra mundial, especialmente en países desarrollados del mundo capitalista.

En la actualidad la ciudadanía juvenil es un tema vigente dado los acelerados cambios políticos, económicos, sociales y culturales, dinamizados por los avances tecnológicos y la globalización de las últimas décadas, en la cual los jóvenes han sido protagonistas dinámicos en diferentes espacios sociales, tanto por su participación como por su exclusión, por encontrarse en una etapa de transición en la que el joven está definiendo su identidad influenciado por la abundante y diversa información a la que accede a través de los nuevos medios de comunicación, desde donde va adoptando e insertando elementos culturales, que se van evidenciando en sus prácticas ciudadanas cotidianas. Este fenómeno es motivo de inquietud entre padres de familia, instituciones académicas y gubernamentales, por lo que pueda suceder con el futuro 
de la sociedad misma, la cual pertenece a los jóvenes de hoy, convirtiéndose en un fenómeno de interés para los académicos, las instituciones sociales y los gobiernos que pueden orientar las políticas públicas sobre juventud.

Es indudable que se están presentando permanentes cambios y de manera acelerada en el entorno juvenil, los cuales buena parte de las veces, no son comprendidos inmediatamente por los demás segmentos sociales, particularmente por los mayores o los de generaciones distintas. Cambios que inquietan, porque entenderlos resulta decisivo teniendo en cuenta que en los jóvenes está la clave del futuro, de valores como la democracia, la libertad o la solidaridad; lo cual implica el mantenimiento del sentido de la organización social, del Estado y las instituciones tal como las conocemos y por las que se ha luchado por más de dos siglos en su consolidación y su defensa (Nussbaum, 2010). Entender a los jóvenes y comprender sus prácticas ciudadanas se convierte en algo clave, porque es a través de la educación desde donde se puede aportar de manera constructiva y acertada en la formación de los ciudadanos que defenderán esos valores, superando las diferencias sociales, económicas y culturales. Por lo anterior es pertinente preguntar: ¿Cuáles son los sentidos o significados de las prácticas ciudadanas de los jóvenes de último grado de educación básica en instituciones educativas de Bogotá? ¿Cuáles son las lógicas de participación de los estudiantes de grado noveno del Colegio Fernando González Ochoa de la localidad de Usme? ¿Cómo perciben los estudiantes del Colegio Fernando González Ochoa las prácticas ciudadanas en el escenario escolar?

Por tanto, el objetivo central de la presente investigación es comprender los sentidos y significados de las prácticas ciudadanas de los jóvenes escolares del último grado de educación básica en instituciones educativas de Bogotá.

\section{Ciudadanía, Cultura escolar y Juventud}

La ciudadanía, la participación juvenil y la cultura escolar — como categorías que se entrelazan permanentemente-, han sido tratadas por diferentes autores e instituciones de orden nacional como internacional, tanto desde el punto de vista 
teórico, histórico, jurídico o como estudios de caso. La Organización de las Naciones Unidas, a través de sus agencias y programas como la Unesco, la Unicef, la OIJ, el PNUD, entre otras, han estudiado el tema de juventud, la participación, la educación ciudadana en el contexto latinoamericano, convirtiéndose en lineamientos para la definición de la política pública sobre juventud en los distintos países. Los Estados miembros de las Naciones Unidas son conscientes de la importancia de los jóvenes como recurso humano fundamental para promover el desarrollo, un cambio social positivo y la innovación tecnológica en cada uno de los países (ONU, 2010). Actualmente, se habla de la educación para la ciudadanía mundial (GCE), este corresponde a uno de los ámbitos estratégicos de trabajo del Programa de Educación de la Unesco (2014-2017) y una de las tres prioridades de la iniciativa mundial: "La educación ante todo", basada en los derechos humanos, la justicia social, la diversidad, la igualdad entre los sexos y la sostenibilidad medioambiental (Unesco, s.f.).

El debate sobre ciudadanía se ha centrado en diversos campos, se logran identificar puntos en común en las diferentes investigaciones, como la importancia dada a la participación juvenil (Balardini, 2000; Fernández, 2012; Flores y Gómez, 2005; Gómez, Torres y Alvarado, 2008), el sentido de pertenencia, la identidad, el reconocimiento de sí mismo y de los otros (Echavarría, 2011; Muñoz y Muñoz, 2008; Sojo, 2002; Vargas, Echavarría, Alvarado y Restrepo, 2007), los derechos y las responsabilidades (Bedoya, Cardona y Gómez, 2010; Chaux, 2012), siendo estas sus principales dimensiones.

\subsection{La ciudadanía, un concepto dinámico}

El concepto de ciudadanía ha sido dinámico y se continúa transformando permanentemente, como consecuencia de los cambios históricos, de las condiciones actuales de la sociedad y de los contextos políticos, económicos, culturales y educativos a los que se enfrentan individual y grupalmente cada segmento generacional de la población. La explicación de este concepto fluctúa a la luz de las diferentes disciplinas con que se le mire - la historia, la sociología, la psicología social, la filosofía, la ciencia política o la economía-, a partir de las cuales se han establecido diferentes 
enfoques y marcos explicativos, con el propósito de evidenciar el ejercicio ciudadano, la participación, la responsabilidad social con lo público, la interrelación con el otro y con el Estado, así como los sentidos de las prácticas sociales cotidianas en cada uno de los segmentos poblacionales, por ende, "la ciudadanía no es algo unívoco, en el sentido de que no se entiende de una única manera” (Miralles, 2009, p. 14).

El concepto mismo de ciudadanía se deriva de ciudad, por las polis —ciudadesestado- griegas y las "civitas" romanas. Durante el renacimiento, en las ciudades italianas, perdura la idea con la consolidación de los estados modernos a partir del siglo XVI y sobre todo con los Estados-Nación de los siglos XIX y XX, el concepto de ciudadanía se vuelve parte de las naciones, organizaciones políticas de las que comenzará a depender la filiación y la garantía de los derechos que concedan dichos Estados. En el mundo globalizado actual se plantea, como realidad o como algo deseable, la ciudadanía transnacional, para casos de comunidades supraestatales como el de la Unión Europea y por otra parte la ciudadanía cosmopolita, referente inevitable, al pensar en una comunidad política mundial (Cortina, 1997).

Para Borja (2001) ser ciudadano o pertenecer a la ciudad "no es solamente "urbs", es decir, concentración física de personas y edificios; es "civitas", lugar del civismo, o participación en los quehaceres públicos; es "polis", lugar de política, de ejercicio de poder" (p. 1). Por ende, "la ciudadanía es la condición de pertenencia y participación en la politeya u organización política donde se integran los miembros de la sociedad " (Moreno, 2003, p. 1). Ser parte de una organización política particular implica una condición legal con beneficios y obligaciones que el ciudadano debe asumir. Así "la ciudadanía es un status, es decir, un reconocimiento social y jurídico por el cual una persona tiene derechos y deberes por su pertenencia a una comunidad, en general, de base territorial y cultural" (Borja, 2001, p. 1). Por tanto, ser ciudadano no tiene que ver solo con los derechos reconocidos por los aparatos estatales a quienes nacieron en un territorio, sino también con las prácticas sociales y culturales que dan sentido de pertenencia y hacen sentir diferentes a quienes poseen una misma lengua, semejantes formas de organizarse y satisfacer sus necesidades (García, 1995). Más que como valores abstractos, los derechos importan como algo que se construye y cambia en relación con prácticas y discursos. Los derechos son 
reconceptualizados, como lo plantea Da Silva (1994), “como principios reguladores de las prácticas sociales, definiendo las reglas de las reciprocidades esperadas en la vida en sociedad a través de la atribución mutuamente acordada (y negociada) de las obligaciones y responsabilidades, garantías y prerrogativas de cada uno" (citado en García, 1995, p. 20).

Sin embargo, del debate sobre ciudadanía surgen diferentes modelos explicativos sobre el concepto desde los años cincuenta del siglo XX, cuando T. H. Marshall (1949) planteó tres tipos de ciudadanía: civil, política y social, de acuerdo con los derechos que se fueron reconociendo desde la Revolución Francesa en los diferentes Estados-Nación del mundo occidental. Para finales de siglo XX, la discusión se centra en los niveles de participación ciudadana en la toma de decisiones sobre lo público, ante la disminución de la participación, evidente en el abstencionismo creciente en las elecciones populares; lo cual permite categorizar la ciudadanía como activa y pasiva (García y Serna, 2002). De igual manera, la ciudadanía es analizada de acuerdo con los modelos políticos, el grado de participación de los ciudadanos y la garantía que se logre en cada comunidad política de la prevalencia de los derechos del individuo (ciudanía liberal), los derechos del grupo (ciudadanía comunitaria) o una intermedia (ciudadanía republicana) para lograr el bien común, para la cual se requiere un alto grado de participación y de nivel cívico (Ochman, 2006).

\subsection{Una mirada a la cultura escolar}

Por su parte la cultura escolar, la cual permite comprender lo que ocurre en el interior de las escuelas, ha adquirido diferentes significados en el campo educativo desde los inicios de su conceptualización, en la década de 1970, hasta el presente, adquiriendo diferentes sentidos teóricos y metodológicos, a partir de los cuales el concepto es construido y utilizado, pero se percibe que aún está inmerso en la ambigüedad. No obstante, las investigaciones realizadas por Schein (1985), Stolp (1994), Huergo (2001), Fernández (2012) y Elías (2015) sobre cultura escolar, permiten analizar de una u otra manera su potencial teórico al brindar herramientas para reconocer el clima escolar en que se llevan a cabo los procesos de enseñanza y aprendizaje y en donde convergen las prácticas ciudadanas. 
Para comprender la cultura escolar es necesario reconocer que los jóvenes llegan a la escuela como sujetos socioculturales, "como individuos que poseen una historicidad, con visiones de mundo, escalas de valores, sentimientos, emociones, deseos, proyectos, con lógicas de comportamientos y hábitos que le son propios" (Dayrell, 1996 p. 141), que convergen con situaciones, relaciones productivas, necesidades, intereses y antagonismos de otros jóvenes, de las familias, los docentes y los administrativos, dentro y fuera de la escuela que sumado a la subjetividad, como la identidad y el cuerpo, la cuestión de raza y de género contribuyen con la construcción o reelaboración de nuevas experiencias culturales a partir de la interacción en la escuela y su entorno (Dayrell, 2010).

El concepto de cultura escolar lo retoma Stolp (1994) como "los patrones de significado transmitidos históricamente y que incluyen las normas, los valores, las creencias, las ceremonias, los rituales, las tradiciones y los mitos comprendidos, quizás en distinto grado, por las personas miembros de la comunidad escolar" (citado en Elías, 2015, p. 278). En otros términos, la cultura escolar es la combinación del conjunto de patrones heredados, inventados, descubiertos o desarrollados que son transmitidos a los nuevos miembros de la comunidad, como la forma correcta de pensar, sentir o comportarse; permeando la forma de actuar, vestir, hablar y de interactuar en la escuela. Lo cual quiere decir que en la cultura escolar están presentes elementos estáticos y dinámicos, siendo la cultura escolar "un conjunto de prácticas, saberes y representaciones producidas y reproducidas a partir de la institución escolar" (Huergo, 2001, p. 4), que llegan a transformar las formas de comunicación e interacción social más allá de la escuela.

\subsection{La juventud. Una categoría cambiante}

La juventud es una categoría construida (Bourdieu, 1990), producto de la manera en que las diversas sociedades perciben y valoran el mundo, y con ello a ciertos actores sociales; para lo cual se construyen categorías como sistema de clasificación de la sociedad, producto del acuerdo social; es por ello que entender el conocimiento que se ha producido con relación a los jóvenes, y reconocer los imaginarios presentes a partir de los cuales se explican diferentes procesos sociales que surgen en determinado 
periodo, son necesarios para pensar y resignificar el papel de estos actores sociales en la actualidad (Reguillo, 2002).

La inserción de los jóvenes a la sociedad hace necesario el establecimiento de procesos que permitan la reivindicación de "la existencia de los niños y los jóvenes, como sujetos de derechos y, especialmente, en el caso de los jóvenes, como sujetos de consumo" (Reguillo, 2002, p. 23); según los planteamientos de esta autora, la ampliación de los rangos de edad son un mecanismo de control social y un dispositivo de autorregulación vinculado a variables como el índice de pobreza, la necesidad de sujetos productivos, el aumento de la expectativa de vida, el desequilibrio en la población económicamente activa, entre otros. Lo anterior se hace evidente en la legislación sobre juventud en Colombia de los últimos veinte años, la cual se modifica así: la Ley 1622 del 29 de abril de 2013 o Ley Estatutaria de Ciudadanía Juvenil establece que joven es toda persona entre 14 y 28 años cumplidos en proceso de consolidación de su autonomía intelectual, física, moral, económica, social y cultural que hace parte de una comunidad política y en ese sentido ejerce su ciudadanía (Ley Estatutaria 1622, 2013). Dicha ley cambió lo establecido por la Ley 375 de julio 4 de 1997 que definía como joven a toda persona entre 14 y 26 años de edad. Adicionalmente, parte del rango de joven coincide con el de adolescente, que es la persona entre los 12 y 18 años de acuerdo con la Ley 1098 de 2006 o Código de la Infancia y la Adolescencia, y según el artículo 34 del Código Civil. Lo anterior evidencia incluso la recategorización de la juventud en diferentes etapas como respuesta y regulación a las inquietudes de cada uno de los segmentos juveniles.

\subsection{La ciudadanía juvenil}

La ciudadanía juvenil se puede considerar como la etapa de transición del joven en la que está desarrollando su autonomía, en la cual es cada vez más consciente de sus derechos, de sus libertades que se van ampliando y de sus restricciones que van disminuyendo; pero sobretodo, de la responsabilidad con la que debe asumir cada una de sus acciones. En ese contexto, la ciudadanía juvenil se manifiesta en las prácticas, en el hacer individual y en las interrelaciones que se generan entre pares a través de actividades artísticas como la música, el baile, la pintura o de cualquier 
otra índole, o simplemente la posibilidad de reunión de manera libre para compartir intereses comunes entre pares; por lo cual "la ciudadanía juvenil, como ciudanía diferenciada, no se refiere exclusivamente al uso de mecanismos de participación ciudadana, sino que involucra sus expresiones, sentires y formas de pensar" (Silva, Cristancho, Parra, Quevedo y Rodríguez, 2015, p. 276). Pensar en la ciudadanía como ejercicio de la política y expresión de lo político demanda nuevas maneras de practicar la política a la luz de las transformaciones históricas y sociales, así como nuevos mecanismos, formas y espacios de organización y participación ciudadana en concordancia con dichas transformaciones; por tanto, la ciudadanía juvenil se puede analizar a partir de dimensiones como: lo jurídico (derechos y deberes), lo territorial (pertenencia e identidad con un territorio) y lo social, cultural y valórico (prácticas y compromiso con una comunidad) Cisternas (2012), donde es importante destacar que las manifestaciones socioculturales como la música, la pintura, el deporte, entre otras manifestaciones artísticas de los jóvenes, son formas de ejercer su ciudadanía, lo cual permite categorizar la ciudadanía juvenil como una ciudadanía cultural (Muñoz y Muñoz, 2008).

\section{Metodología}

La investigación es de tipo cualitativo con enfoque hermenéutico-interpretativo, busca comprender y profundizar los fenómenos sociales, explorándolos desde la perspectiva de los participantes en un ambiente natural y en relación con el contexto (Hernández, Fernández y Baptista, 2010). La investigación se basa en la observación directa de un fenómeno social por parte del investigador, por tanto participante, donde se describen, analizan y en algunos casos se interviene, dado el rol del investigador-docente, sobre las prácticas ciudadanas de una población escolar juvenil, en un espacio institucional, en este caso los jóvenes de grado noveno del Colegio Fernando González Ochoa ubicado en la localidad de Usme en Bogotá D. C.

Para la recolección de la información en el presente trabajo se utilizaron principalmente las siguientes técnicas de investigación: observación participante, realizada por el docente-investigador, profesor del área de Ciencias Sociales durante los últimos tres años en la institución (presente en el aula, en espacios comunes y en 
actividades institucionales); grupos focales, dos experiencias con 6 y 7 participantes respectivamente, compuestos por hombres y mujeres, con nivel académico diverso y un afrodescendiente en cada uno; y entrevistas semiestructuradas a dos docentes que conocían a los estudiantes objeto de estudio y su contexto social Es importante señalar que previa a la aplicación de dichas herramientas de investigación, se realizaron sondeos exploratorios a los estudiantes, sobre nociones de ciudadanía, lo cual permitió orientar las observaciones y la formulación de las preguntas para cada instrumento de investigación.

\section{RESULTADOS Y DISCUSIÓN. LAS PRÁCTICAS CIUDADANAS EN LA ESCUELA}

La conformación poblacional de sectores periféricos populares de ciudades como Bogotá, propio del caso de estudio, a donde llegan migrantes de todas las regiones de Colombia, tanto de áreas urbanas como rurales, de condiciones sociales, económicas, étnicas y culturales diferentes, incluyendo a algunos desplazados por la violencia del conflicto armado colombiano, hace de la escuela y de estos territorios un espacio "multicultural" en donde se aprende a comprender, o por lo menos a respetar, las maneras de pensar y de actuar del otro, y en muchos casos a generar sentimientos de solidaridad y apoyo que incluso llega hasta las familias de los estudiantes. Considerando que el colegio queda cerca de la frontera rural-urbana y que gran parte de los habitantes de la zona provienen de áreas rurales, es válida la percepción de un docente cuando afirma que los estudiantes del colegio Fernando González Ochoa “a nivel cultural todavía responden a unas prácticas enmarcadas en lo popular, [...], unos patrones rurales de comportamiento, tanto de los padres como de ciertos muchachos...".

A partir del sondeo exploratorio, se les preguntó a 37 estudiantes de entre 14 y 17 años: ¿Usted se considera un ciudadano? Sí. No, ¿por qué? ¿Qué entiende por ciudadanía? ¿Cómo considera que se ejerce la ciudadanía? ¿Qué considera (hechos, espacios o situaciones) que facilita el ejercicio de la ciudadanía? ¿Qué considera (hechos, espacios o situaciones) que limita el ejercicio de la ciudadanía? La mayoría de 
los consultados se reconocen como ciudadanos, se consideran parte de un territorio, pertenecientes a una organización política, como lo es la ciudad, a una comunidad barrial o zonal dentro de la misma ciudad, que les da unos derechos y que también les acarrea cumplir con unos deberes; aunque en las respuestas se evidencian diferencias o matices, es coherente si se tiene en cuenta que hay estudiantes de distintos lugares de Colombia y por tanto han tenido experiencias de vida diversas.

Por el contrario, una pequeña minoría de jóvenes no se consideran ciudadanos ya que no se sienten escuchados o tenidos en cuenta en la toma de decisiones en sus comunidades o barrios, a ello se suma el hecho de no tener derecho a sufragar en las elecciones políticas debido a su minoría de edad. A su participación restringida se agrega la dependencia que tienen de padres o tutores y de docentes en la institución escolar, e incluso del Estado, y con la que algunos estudiantes juegan según las circunstancias, "...tengo derecho (...) porque soy menor de edad" o "es que a mí no me pueden hacer nada porque soy menor de edad", esto lo asocian además con que no son ciudadanos con plenos derechos, porque aún no tienen la cédula de ciudadanía, que será el momento en el que ellos podrán hacer lo que quieran y serán directa y legalmente responsables por lo que hagan.

Sin embargo, y a pesar de las dudas generadas incluso en algunos docentes, la mayoría de jóvenes tienen una conciencia de su papel en la sociedad y como parte integrante del Estado, quien reconoce sus derechos y deberes, aunque sea de manera superficial y algunas veces juega con la prevalencia que la ley les da por ser menores de edad; hacen comentarios de las tutelas para acceder a derechos como la salud y la educación por situaciones conocidas de familiares, vecinos, compañeros, reportajes o noticias de radio y televisión, que si bien no todos ven o escuchan directamente del medio, es socializado en la escuela por el voz a voz o a través de las redes sociales. Al respecto, a continuación parte de la respuesta de un estudiante participante de un grupo focal frente a la pregunta, ¿conoce sus derechos y sus deberes?, ¿los cumple?: "conocemos nuestros deberes y derechos, buscamos que se nos respeten nuestros derechos, buscamos no cumplir nuestros deberes bien sea por pereza o porque voy de afán, o por influencia de otros que tampoco cumplen, o por lo que sea”. Sin embargo, los estudiantes tienen claro que muy pronto cumplirán la mayoría de edad, podrán 
sufragar y ejercer como ciudadanos plenos, y que por lo tanto también recaerá toda la responsabilidad civil sobre ellos.

Por diversos canales, situaciones, vivencias o socialización entre ellos, la mayoría de jóvenes tienen conocimiento de la existencia de las múltiples instituciones legales como personerías municipales y locales, defensoría del pueblo, juzgados de familia, juzgados de menores, bienestar familiar, fundaciones y centros de rehabilitación; lugares donde desde diferentes puntos de vista se tratan los derechos del menor, lo cual ha permitido un alto nivel de apropiación de los elementos legales sobre el tema de niñez y adolescencia, particularmente sobre protección. Aunque son desafortunadas muchas de las situaciones que han tenido que vivir algunos de estos jóvenes y por las cuales llegaron a conocer dichas instituciones, es positivo el restablecimiento de derechos a la población en estado de vulnerabilidad, sin embargo, algunos estudiantes tratan de manipular la ley a su conveniencia dentro del colegio para justificar el incumplimiento de algunas normas o deberes.

La apropiación de la norma se evidencia nuevamente respecto a los derechos sexuales y reproductivos, a partir de casos conocidos difundidos por los medios de comunicación y redes sociales. Desde agosto de 2014 y durante 2015 y parte de 2016 fue ampliamente comentado, en diferentes medios de comunicación, el caso del estudiante Sergio Urrego que se suicidó por presunta discriminación, debido a su orientación sexual. Este caso motivó diferentes pronunciamientos de la ministra de Educación y que se plantearan proyectos de ley sobre el tema, así como cartillas sobre derechos sexuales y reproductivos que terminaron siendo muy polémicas socialmente. Estos jóvenes utilizan casos como el de Urrego para justificar algunas conductas entre parejas de novios como besos apasionados o determinadas caricias que limitan con lo erótico y que son realizadas en público, durante la hora de descanso e incluso en el aula, que ellos defienden como actos afectivos a los que tienen derecho. Estas situaciones terminan siendo manejadas según el criterio del docente, dado que este tema, tanto en el manual de convivencia como legalmente, es confuso y se presta para múltiples interpretaciones o resulta muy laxo y es complicado determinar dónde termina y comienza un simple acto afectivo, un episodio morboso o de exhibición que vaya en contra de las "buenas costumbres" o lo socialmente aceptado como correcto o debido. 
En general, los jóvenes saben sacar beneficio de la prevalencia de los derechos legalmente establecidos, a partir de lo cual adaptan sus prácticas ciudadanas en la escuela, buscando mantenerse en el límite, entre lo permitido y no permitido, en la relación con sus pares, con los docentes y administrativos, así como con el cuidado de la planta física y el mobiliario del colegio; buscando desarrollar actividades de su agrado, tanto grupal como individualmente, lo cual se evidencia en las expresiones artísticas (música, RAP, grafiti, dibujo, etc.) o recreativas (microfútbol, la lleva, ajedrez entre otros) tanto en actividades institucionales o no institucionales, en las que se puede ver un importante nivel de sensibilidad, solidaridad y cumplimiento del deber con aquello que les interesa o afecta directamente.

\subsection{La participación en la escuela}

A partir de la observación de las prácticas ciudadanas de los estudiantes en el entorno escolar y la participación juvenil en la escuela, en la presente investigación se categoriza en participación formal y no formal. La participación formal se asocia con las propuestas institucionales con espacios y reglas de participación establecidas, versus la participación informal o no formal que no es planteada por instituciones y puede surgir en cualquier espacio (esquina, rincón, pared) a partir de grupos e intereses espontáneos. A la primera corresponden todas las actividades o celebraciones asociadas con jornadas académico-culturales de proyectos de ley, transversales o institucionales, que hacen parte de actividades pedagógicas lideradas por docentes de diferentes áreas, como ocurre con el gobierno escolar, el english day, la colombianidad, las izadas de bandera, la semana por la paz, entre otros; temas que los estudiantes no eligieron, porque ya estaban definidos y por los que normalmente esperan una valoración académica. Por otro lado, la participación no formal se relaciona con iniciativas espontáneas, definidas por los mismos grupos de estudiantes, de cursos diferentes, alrededor de intereses comunes como la música, el deporte, el arte, las creencias religiosas o en actividades de esparcimiento basadas en relaciones de amistad o empatía, dando origen a colectivos juveniles básicos, algunos de los cuales trascienden la escuela y permanecen por tiempos prolongados y que se establecen sin esperar ninguna recompensa de tipo académico. 
No se observan iniciativas juveniles alrededor de temas como lo ambiental, la diversidad sexual y de género, lo étnico, lo económico ni lo político porque en el contexto social son problemáticas a las que no se les ha dado mayor trascendencia, o porque consideran por fuera de sus responsabilidades de jóvenes. Además de ello no ven la aplicabilidad en su cotidianidad manifestando poco interés sobre estos temas.

\section{Conclusiones}

Las prácticas ciudadanas de jóvenes de último grado de educación básica del colegio Fernando González Ochoa de la localidad de Usme, se encuentran enmarcadas en un sector popular de la ciudad e influenciadas por elementos rurales, de una población con un alto porcentaje de migrantes de áreas rurales y urbanas de diferentes regiones del país, entre los que hay emigrantes económicos que buscan mejores oportunidades de vida y desplazados forzados por el conflicto armado colombiano. Adicionalmente, en estas familias predominan bajos niveles de escolaridad y algunas han tenido experiencias traumáticas de violencia, otras, dificultades económicas extremas, así como casos de desintegración o recomposición familiar, lo cual ha incidido de diferentes maneras en las prácticas ciudadanas y comportamientos de los jóvenes en el entorno escolar.

Lo anterior permite que una institución educativa como la analizada cuente con una gran diversidad social y cultural, que sumada a las múltiples experiencias tanto de los estudiantes como de sus familias, hace de estos jóvenes sujetos con un relativo sentido de tolerancia por las diferentes maneras de pensar y actuar de los demás; es decir, que mientras no sean afectados o se sientan violentados en algún grado, de manera directa o indirecta tienden a ser indiferentes, en caso contrario suelen responder de manera violenta, sin pensar o medir las consecuencias, como la forma típica que conocen de resolver los conflictos.

Por otro lado, las diferentes vivencias de algunos jóvenes generan sentido de identidad con pares en situaciones similares, lo que conlleva a fortalecer el valor de la solidaridad por el que se encuentra mal económica, familiar o afectivamente, y se evidencia 
en las expresiones de hermandad, algunos literalmente se tratan de "hermano", y comparten prendas de vestir, útiles escolares e incluso su propia comida, además de la parte afectiva que permite llenar vacíos psicoafectivos. Son muy sensibles con las situaciones de injusticia generalizada que perciben en su entorno, en la ciudad y en el país en general, porque la han vivido directa o indirectamente a través de familiares o personas cercanas.

$\mathrm{Al}$ analizar cómo perciben la escuela en Bogotá, los jóvenes están de acuerdo en que este es un espacio de encuentro para compartir saberes, gustos musicales, actividades artísticas o deportivas con los compañeros o amigos en condiciones seguras. Que la escuela es el lugar de interacción social donde se establecen y cultivan lazos de amistad. Todo lo anterior se convierte para los jóvenes en una de las principales motivaciones para asistir a la escuela a pesar de que ciertas actividades académicas o asignaturas no sean de su agrado, el compartir con los demás siempre será importante.

Al confrontar las diferentes fuentes se concluye que los jóvenes escolares durante el nuevo milenio, si bien hacen escaso uso de los medios tradicionales de información como la prensa escrita en físico o la radio para informarse, utilizan algunas de las múltiples posibilidades a través de los medios digitales, donde la información se presenta multimedialmente, de manera más dinámica, haciéndola más atractiva para ellos, aunque dicha información no siempre se presente con la suficiente profundidad o induzca a lecturas parcializadas. La información que llama la atención a los jóvenes, normalmente es compartida en redes sociales como Facebook, WhatsApp o Twitter, con los contactos, amigos y conocidos. Este hecho hace que las redes sociales se conviertan para muchos jóvenes en el principal medio de comunicación y de consumo.

Los jóvenes durante el proceso de interacción con sus pares y como producto de toda la información que reciben y apropian a través de los medios de comunicación y las redes sociales, construyen y ajustan, en la medida de sus necesidades y posibilidades, sus propias reglas o normas de comportamiento, que regulan las relaciones entre ellos y además se convierte en una forma diferente de interpretar los códigos establecidos por los adultos. Esto conlleva a que dichas normas suelan ser contrarias a los modelos 
tradicionales y consideradas por los adultos como parte de la rebeldía juvenil o como comportamientos inmorales; sin embargo, diferentes autores reivindican estas actitudes juveniles como la forma de evolución y de cambio en las relaciones y en los sistemas de valores sociales, culturales y políticos, lo cual no implica que sean menos buenos que los vigentes, pero a los cuales los adultos suelen ver con desconfianza e incluso sin futuro, porque rompe con esquemas sociales y culturales preestablecidos.

Respecto al concepto de ciudadanía y el ejercicio ciudadano, y de acuerdo con Cisternas (2012), el discurso de la gran mayoría de los jóvenes se enmarca en una concepción tradicional y convencional relacionada con el sistema normativo de derechos y deberes otorgados por el Estado; sin embargo, también se identifican otras concepciones que con la anterior se pueden clasificar en: la ciudadanía jurídico política, la ciudadanía territorial y por último la ciudadanía social, cultural y valórica, relacionada con las prácticas sociales comunitarias y cotidianas, en el que el aprender a conocer, aprender a hacer, aprender a vivir juntos y aprender a ser, son fundamentales para el desarrollo del nuevo ciudadano. Es por ello que las manifestaciones socioculturales, a partir de las cuales grupos excluidos socialmente o vulnerables ejercen su ciudadanía, adquieren un gran valor e importancia, es decir, lograr ejercer la ciudadanía juvenil como una ciudadanía cultural, tal como lo plantean Muñoz y Muñoz (2008), lo cual abarca las dinámicas sociales juveniles como: sus modos y formas de comprender la vida y sus adscripciones identitarias, relacionado con la música, las expresiones artísticas y culturales, las posibilidades de consumo y las diferentes formas de habitar, comprender la ciudad y sus propios cuerpos como primer territorio. Es mediante esas prácticas que los jóvenes realmente se revelan como sujetos ciudadanos capaces de construir nuevos códigos, nuevas formas de expresar sus sensibilidades y de diferenciarse de los adultos como una manera de resistencia. 


\section{REFERENCIAS BIBLIOGRÁFICAS}

Balardini, S. (Ed.). (2000). La participación social y política de los jóvenes en el horizonte del nuevo siglo. Buenos Aires, Argentina: CLACSO, Consejo Latinoamericano de Ciencias Sociales. Recuperado de http://bibliotecavirtual.clacso.org.ar/ar/libros/cyg/juventud/juventud.html

Bedoya, D., Cardona, B., y Gómez, P. (2010). Configuración del sentido de responsabilidad del sujeto joven en el espacio escolar. Medellín, Colombia: Universidad de Manizales CINDE. Recuperado de http://ridum.umanizales.edu.co:8080/xmlui/bitstream/handle/6789/1374/Bedoya_Gutierrez_Diana_Patricia_2010.pdf?sequence=1

Borja, J. (junio de 2001). La ciudad y la nueva ciudadanía. Conferencia pronunciada en el «Fórum Europa». Barcelona. Recuperado de http://www.diba.cat/forumeuropa/documents_2001/Borja.doc

Bourdieu, P. (1990). Sociología y Cultura. México: Editorial Grijalbo.

Chaux, E. (2012). Educación, convivencia y agresión escolar. Bogotá D. C.: Universidad de Los Andes.

Cisternas, L. E. (2012). Las representaciones de ciudadanía en jóvenes secundarios chilenos construcción de ciudadanía juvenil. Santiago, Chile: Universidad de Chile.

Cortina, A. (1997). Ciudadanos del mundo. Hacia una teoría de la ciudadanía. Madrid: Alianza Editorial.

Dayrell, J. (1996). A escola como espaço sócio-cultural. Ensino de Sociologia. Retrieved from http://ensinosociologia.pimentalab.net/files/2010/09/Dayrell-1996-Escola-espaço-socio-cultural.pdf

Dayrell, J. (2010). Juventud, socialización y escuela. Archivos de Ciencias de la Educación, $4(4), 15-34$. 
Echavarría, C. V. (2011). Concepciones de ciudadanía y de ejercicio ciudadano en un grupo de jóvenes en situación de protección. Revista Educación y Pedagogía, 23(59), 197-212.

Elías, M. E. (2015). La cultura escolar: aproximación a un concepto complejo. Revista Electrónica Educare, 19(2), 285-301. Recuperado de http://www.revistas.una.ac.cr/index. php/EDUCARE/article/view/6591/6693

Fernández, C. (2012). Ciudadanía juvenil y nuevas formas de participación a través de la conectividad. Culturales, 8(15), 113-134. Recuperado de http://www.scielo.org.mx/scielo. php?script=sci_arttext $\&$ pid $=$ S1870-11912012000100005

Flores, P., y Gómez, N. R. (2005). Ciudadanía juvenil: sin espacios, ¿¿dónde construirla ? Investigación y Desarrollo, 13(1), 78-107. Recuperado de http://rcientificas.uninorte.edu. co/index.php/investigacion/article/view/1064/657

García, N. (1995). Consumidores y ciudadanos o el consumo como hipérbole. Revista Austral Ciencias Sociales, (2), 131-133. México: Editorial Grijalbo. Recuperado de http:// doi.org/10.4206/rev.austral.cienc.soc.1998.n2-14

García, R., y Serna, A. (2002). Dimensiones críticas de lo ciudadano. Esbozo para un estado del arte sobre ciudadanía. Dimensiones críticas de lo ciudadano: problemas y desafios para la definición de la ciudadanía en el mundo contemporáneo, 1-33. Bogotá D. C., Colombia: Universidad Distrital Francisco José de Caldas.

Gómez, P. B., Torres, J., y Alvarado, S. V. (2008). Perspectivas teóricas para comprender la categoría participación ciudadana-política juvenil en Colombia. Revista Latinoamericana de Ciencias Sociales, Niñez y Juventud, 6(2), 565-611. Recuperado de http://www.scielo. org.co/scielo.php?pid=S1692-715X2008000200005\&script=sci_abstract\&tlnges

Hernández, R., Fernández, C., y Baptista, M. P. (2010). Metodología de la investigación. México: McGraw-Hill. 
Huergo, P. J. A. (2001). Desbordes entre la cultura escolar y la cultura mediática. Revista Nómadas, 15, 88-100. Recuperado de http://dialnet.unirioja.es/servlet/ articulo? codigo $=3990699$

Ley Estatutaria 1622. (2013). Por medio de la cual se expide el estatuto de ciudadania juvenily se dictan otras disposiciones. Diario Oficial n. ${ }^{\circ}$ 48.776. Recuperado de https://www.icbf. gov.co/cargues/avance/docs/ley_1622_2013.htm

Marshall, T. H. (1997). Ciudadanía y clase social. REIS. Revista Española de Investigaciones Sociológicas, 79, 297-344. Recuperado de http:/www.reis.cis.es/REIS/ jsp/REIS.jsp?opcion=articulo\&ktitulo=1288\&autor=THOMAS+HUMPHREY+MAR SHALL

Miralles, J. (2009). Sobre el concepto de ciudadanía: historia y modelos. Factótum, 1-22. Recuperado de http://dialnet.unirioja.es/servlet/articulo?codigo=3140837\&orden=237 $327 \&$ info=link

Moreno, L. (2003). Ciudadanía, desigualdad social y Estado del bienestar. Documento de trabajo 03-08. Recuperado de http://digital.csic.es/bitstream/10261/1570/1/dt-0308.pdf

Muñoz, G., y Muñoz, D. A. (2008). La ciudadanía juvenil como ciudadanía cultural: una aproximación teórica desde los estudios Culturales. Revista Argentina de Sociología, 6(11), 217-236. Recuperado a partir de http://www.scielo.org.ar/pdf/ras/v6n11/v6n11a11.pdf

Nussbaum, M. (2010). Sin fines de lucro. Por qué la democracia necesita de las humanidades. Madrid: Katz Editores.

Ochman, M. (2006). Alternativas teóricas de la participación ciudadana en las decisiones públicas. Ciudadanos, Decisiones Politicas y Calidad de la Democracia. México: Limusa.

ONU. (2010). Año Internacional de la Juventud. New York, Estados Unidos. Recuperado de http://social.un.org/youthyear/docs/brochure-sp.pdf 
Reguillo, R. (2002). Emergencia de culturas juveniles. Estrategias del desencanto. Revista Mexicana de Sociología, 64(2), 149. http://doi.org/10.2307/3541499

Schein, E. H. (1992). Organizational Culture and Leadership. San Francisco, CA.: Jossey-Bass.

Silva, S., Cristancho, L., Parra, C., Quevedo, N., y Rodríguez, A. (2015). Ciudadanía Juvenil: una breve revisión. Diversitas-Perspectivas en Psicología, 11(2), 273-288.

Stolp, S. (1994). Liderazgo para la cultura escolar. Centro de información sobre política y gestión educativa. Recuperado de http://www.ericdigests.org/2003-3/escolar.htm

Sojo, C. (2002). La noción de ciudadanía en el debate latinoamericano. Revista de la CEPAL, 76, 25-38. Recuperado de http://repositorio.cepal.org/bitstream/handle/11362/ 10799/076025038_es.pdf?sequence=1

Unesco. (s.f.). La educación para la ciudadanía mundial (GCE). Recuperado de http://www. unesco.org/new/es/education/resources/in-focus-articles/global-citizenship-education/

Vargas, H. H., Echavarría, C. V., Alvarado, S. V., y Restrepo, J. A. (2007). Sentidos de ciudadanía en un grupo de jóvenes escolarizados de la ciudad de Manizales, Colombia. Latinoamericana de Ciencias Sociales, Niñez y Juventud, 5(2), 691-721. Recuperado de http://www.scielo.org.co/pdf/rlcs/v5n2/v5n2a07.pdf 